\title{
Multicriterial optimization of preventive maintenance of informational/technical stochastic system
}

\author{
Anna Kovalenko ${ }^{1, a}$ and Gennady Rogachev ${ }^{1}$ \\ ${ }^{1}$ Samara State Technical University, 443100, Samara, Russia
}

\begin{abstract}
The problem of optimization of reliability and economical indexes is solved for informational/technical system with periodical diagnostics and restoration. The system considered is the one with users accesses and errors occurring. The probability that the error occurs increases in time. Therefore, the longer is the restoration period the more there is a risk of system operation with error. But too frequent restoration can be too expensive. To take into account all these factors two-criteria optimization is made with the restriction of reliability characteristic being of the given level.
\end{abstract}

\section{Introduction}

It is impossible to imagine the modern enterprise operating without informational/technical systems of various complexity and reliability. The diagnostics of such systems has gained particular significance [1]. Many authors apply queue models to model informational/ technical systems and get their adequate economical and reliability characteristics. Herewith, one should take into account their breakdowns, like in [2].

The problem of system optimization is often solved at the stage of system projection. One of the efficient solutions is planning diagnostics of the system. As the continuous diagnostics of informational/technical systems is expensive, they often apply the strategy of periodical diagnostics.

The system considered is the one with users accesses and errors occurring. The probability that the error occurs increases in time. Therefore, the longer is the restoration period the more there is a risk of system operation with error. But too frequent restoration can be too expensive. To take into account all these factors two-criteria optimization is made with the restriction of reliability characteristic being of the given level.

The system is considered to be stochastic. More specifically, we construct the model of one-server queue with immediate service and periodic diagnostics. To get system stationary characteristics the theory of regenerative processes is applied. To simplify the models they often take random variables to have exponential distributions (for example, [3]). We do not make these restrictions.

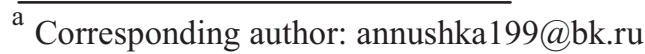

\section{Problem setting}

Let us consider informational/technical system with periodical diagnostics and restoration. Herewith, the system is down while restoration. The system operates in the following way.

Time period between users accesses is the random variable $\beta$ with the distribution function $G(t)=P\{\beta \leq t\}$ and density $g(t)$. Diagnostics period is $\tau$. The function $p(\tau)$, depending on the diagnostics period, is the probability that there is no error and the system continues to operate. The function $q(\tau)=1-p(\tau)$ denotes the probability the error is found and the system restoration is begun. Restoration time is proportional to $\tau$. The proportionality coefficient is $k$. The random variables $\beta$ is supposed to be independent, to have absolutely continuous distribution function and finite expectation $M \beta$.

\section{Mathematical model building}

To describe the system operation one can apply semiMarkov process with a discrete-continuous phase state space [4]. It was done in [5, 6], for instance. But as the process describing the system is regenerative, system stationary characteristics can also be obtained another way. System operation time diagram is represented in Figure 1.

With the help of Fig. 1 one can obtain final probabilities of system restoration and normal operation respectively. As we get stationary characteristics it is enough to consider one regeneration period. In addition, 
we can do it by writing out geometrical probabilities of the system states.

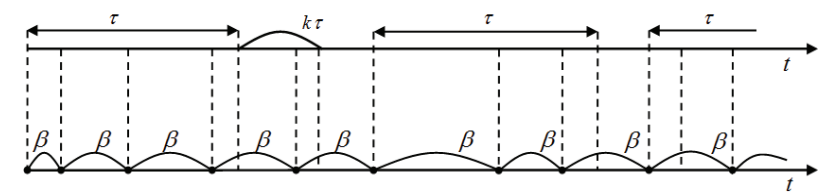

Figure 1. System operation time diagram.

If $p_{0}(\tau)$ is the final probability the system is under restoration, $p_{1}(\tau)$ is the final probability the system operates, then

$$
p_{0}(\tau)=\frac{k q(\tau) \tau}{M \beta p(\tau)\left[1+H_{g}(\tau)\right]+q(\tau) M \beta\left[1+H_{g}(\tau+k \tau)\right]}
$$

$$
p_{1}(\tau)=1-\frac{k q(\tau) \tau}{M \beta p(\tau)\left[1+H_{g}(\tau)\right]+q(\tau) M \beta\left[1+H_{g}(\tau+k \tau)\right]}
$$

In the same way, system economical indexes, such as the average specific income $S(\tau)$ per calendar time unit and average specific cost $C(\tau)$ per time unit of normal operation are:

$$
S(\tau)=\frac{S_{1}(\tau) p(\tau)\left[1+H_{g}(\tau)\right]-c_{0} k q(\tau) \tau-C_{2}}{M \beta p(\tau)\left[1+H_{g}(\tau)\right]+q(\tau) M \beta\left[1+H_{g}(\tau+k \tau)\right]},
$$

$$
C(\tau)=\frac{c_{0} k q(\tau) \tau+C_{2}}{M \beta p(\tau)\left[1+H_{g}(\tau)\right]+q(\tau) M \beta\left[1+H_{g}(\tau+k \tau)\right]-k q(\tau) \tau}
$$

Here, $S_{1}(\tau)$ is the income per successive user access to the system decreasing with time as the probability of errors absence decreases: $S_{1}(\tau)=S_{1} p(\tau), \quad S_{1}$ is the income per successive user access to the system without errors; $c_{0}$ is the cost per time unit of system restoration; $C_{2}$ is the cost of one diagnostics, $H_{g}(\tau)$ is the renewal function of the renewal process generated by the random variable $\beta$.

For the system where $g(t)=\lambda e^{-\lambda t}$, $p(\tau)=\mu /(\mu+\tau), \tau \geq 0$, formulas (1) and (2) take the form:

$$
\begin{gathered}
p_{0}(\tau)=\frac{k \lambda \tau^{2}}{\lambda(1+k) \tau^{2}+(1+\lambda \mu) \tau+\mu}, \\
p_{1}(\tau)=\frac{\lambda \tau^{2}+(1+\lambda \mu) \tau+\mu}{\lambda(1+k) \tau^{2}+(1+\lambda \mu) \tau+\mu} \\
S(\tau)=\lambda \frac{\left(S_{1} \lambda \mu-C_{2}\right) \tau-c_{0} k \tau^{2}+\mu\left(S_{1}-C_{2}\right)}{\lambda(1+k) \tau^{2}+(1+\lambda \mu) \tau+\mu}, \\
C(\tau)=\lambda \frac{c_{0} k \tau^{2}+C_{2} \tau+C_{2} \mu}{\lambda \tau^{2}+(1+\lambda \mu) \tau+\mu} .
\end{gathered}
$$

\section{Optimal period of system restoration}

The following criteria of system optimal operation are taken:
1) positive criterion - average specific income per calendar time unit $S(\tau)$;

2) negative criterion - average specific cost per time unit of normal operation $C(\tau)$;

There is also a restriction given by the inequality for the probability of normal system operation $p(\tau)$.

$$
\left\{\begin{array}{l}
S(\tau) \rightarrow \max _{\tau \in(0, \infty)}, \\
C(\tau) \rightarrow \min _{\tau \in(0, \infty)}, \\
p(\tau)>\widetilde{P} .
\end{array}\right.
$$

One of the methods of multi-criteria problem reduction to one-criteria is to take linear convolution of criteria as objective function [7]. In our case, economical indexes are of the same nature, that is why the objective goal is $V(\tau)$ :

$$
V(\tau)=a_{s} S(\tau)-\left(1-a_{s}\right) C(\tau) .
$$

Here, $a_{s}$ is a weight coefficient defining the indexes of relative significance of $S(\tau)$. The function $p(\tau)$ is monotonously decreasing over the positive values of its argument. That is why in the case considered the optimization problem is the problem of obtaining the value $\tau_{o p t}^{V}$ of absolute maximum of the function $V(\tau)$ at $\tau \in\left(0, \tau_{p}\right), p\left(\tau_{p}\right)=\widetilde{P}$.

Consider an example of optimization of diagnostics period. Inter-arrival time is exponential, the average time between users accesses is 2,5 hours; $p(\tau)=1 /(1+0.05 \tau), \tau \geq 0 \quad k=0,2 ; \quad \widetilde{P}=0,8 ;$ $S_{1}=1000$ money units (m.u.), $c_{0}=400$ m.u./hour, $C_{2}=350$ m.u.

The solution of the problem by means of Maple application gives the following results. The linear convolution of criteria, if the weight coefficient is $a_{s}=1 / 2$, reaches its highest value, satisfying the inequality restrictions at the point $\tau_{o p t}^{V}=3,21$ hours: $S\left(\tau_{o p t}^{V}\right)=219,92$ m.u./hour, $\quad C\left(\tau_{o p t}^{V}\right)=67,52$ m.u./hour, $p_{1}\left(\tau_{\text {opt }}^{V}\right)=0,985, p_{1}\left(\tau_{\text {opt }}^{V}\right)=0,862$. Graphs of reliability criterion (the probability of system normal operation) against diagnostics period is given in Fig. 2. Graphs of economical criteria against diagnostics period are given in Fig. 3, 4. Graph of linear convolution of economical criteria against diagnostics period is given in Fig. 5 .

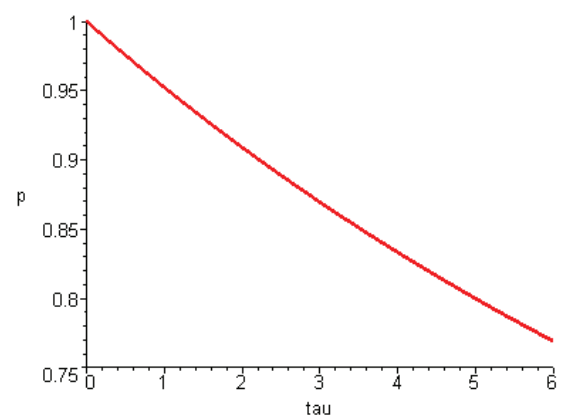

Figure 2. Graph of final probability of normal system operation against diagnostics period. 


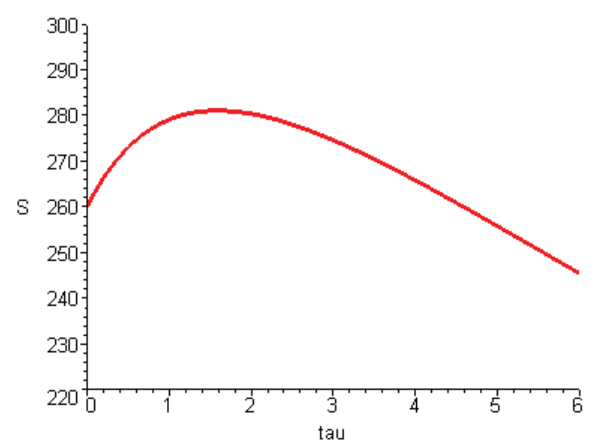

Figure 3. Graph of average specific income per calendar unit against diagnostics period.

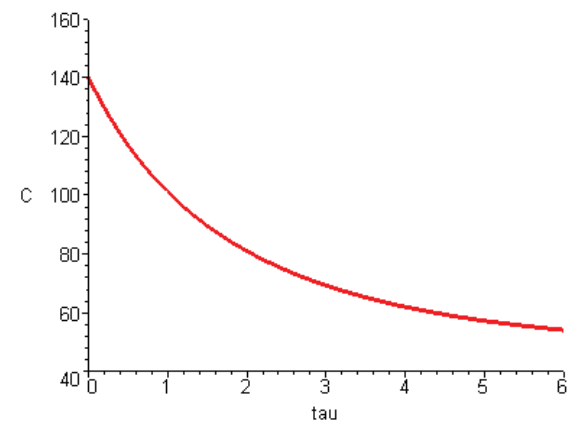

Figure 4. Graph of average specific cost per time unit of normal operation against diagnostics period.

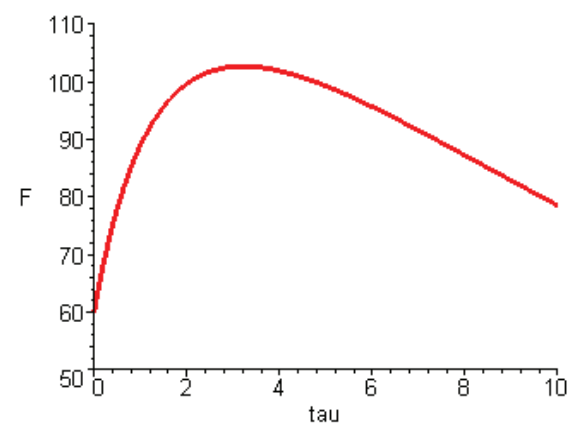

Figure 5. Graph of linear convolution of criteria against diagnostics period.

\section{Acknowledgment}

This work was financially supported by Ministry of Education and Science of Russian Federation (basic part of government contract, project No. 1271).

\section{References}

1. Ya. A. Maksimov. 8 International conference on mathematical modelling and computer science YM2007 (2007) [Rus]

2. U. Yechiali. Queueing Systems, 56, 195 (2007)

3. N. Perel, U. Yechiali. EJOR, 201, 247 (2010)

4. V.S. Korolyuk, A.F. Turbin. Markov Renewal Processes in System Reliability Problems (Nauka, 1982) [Rus]

5. A.I. Peschansky, A.I. Kovalenko. Cybernetics and Systems Analysis, 51, 632 (2015)
6. Yu. Obzherin, El. Boyko. Semi-Markov models. Control of restorable systems with latent failures (Elsevier, 2015)

7. M. Emmerich, A. Deutz. Multicriteria Optimization and Decision Making (Springer, 2006) 